physiology of normal man and the application of its results in industry and the services. A number of interesting and varied projects are currently in progress at the laboratories. In the Department of Human Biomechanics, for example, R. J. Whitney is studying movements of normal people. Photography is used for an exact analysis of the motion of different parts of the body, and the presence or absence of activity in specific muscles which may be involved in the motion is registered by electromyography. One experiment, completed nine months ago, was concerned with the effects of prohibition of movement on physiological and psychological activities of man. Several subjects under thirty were examined in four types of sitting positions varying from supine to upright during twenty-four hours of confinement. Among the changes recorded were EMG of limbs, heart pulse and posture change. A time lapse film indicated that principal movement of the head and feet occurred during the twenty-four hours. Although the results are difficult to analyse, there is evidence of a definite diurnal rhythm in temperature and in swelling of the ankles.

The function of the Division of Biomedical Engineering is to establish a link between modern technology and human biology. It has designed SAMI (Socially Acceptable Monitoring Instrument) to investigate "normal man leading a normal life". This heart beat counter, which derives its input signal from two adhesive chest electrodes, can store acquired information for prolonged periods by means of a reversible electrochemical integrator. Using SAMI, emotional stress has been measured in airline pilots, pregnant women, Peruvian women and schoolchildren. SAMI can also be used to estimate human activity and in clinical follow-up and in work study. A temperature SAMI is also becoming available and SAMIs for noise and posture are in preparation. The development of FAIR (Fast Access Information Retrieval) in the department should lead to full-text, special subject collections becoming available on a user's desk, together with a retrieval system which allows one to generate highly specific subject headings. By providing a list of key words for coding documents, it is hoped that a larger scientific population will consult literature sources of information.

Another interesting study at the laboratories is the investigation of anaemia during pregnancy in immigrant mothers with abnormal haemoglobins and thalassaemia. In the heterozygous conditions, these are both clinically mild but become more severe during increased physiological stress. The traits can be identified by starch-gel electrophoresis and treated with iron administered orally. In 1966 a Tristan da Cunha working party was set up through the council, to investigate the health of the islanders and to assess the balance of their diets. Results indicated that the islanders do not, in fact, live up to their reputation for good health.

\section{Food Additives}

THE use of food additives is difficult to control satisfactorily, for although many countries have agencies and scientific institutions with this special responsibility, others lack adequate facilities. The problem is being dealt with by a joint $\mathrm{FAO} / \mathrm{WHO}$ programme which aims to make systematic evaluations of food additives and to provide evidence to member states of FAO and WHO. The programme is implemented by the joint $\mathrm{FAO} / \mathrm{WHO}$ Expert Committee on Food Additives which advises the Codex Committee on Food Additives, which in turn, through the Codex Alimentarius Commission, proposes to governments internationally acceptable tolerances for additives in various foods.

The Codex Committee selects the substances to be considered by the Expert Committee, whose terms of reference are to establish specifications for identity and purity for food additives and to evaluate toxicological data. It also recommends, if possible, acceptable daily intakes for man. This committee has just produced its tenth report, on "Some Emulsifiers and Stabilizers and Certain Other Substances" (WHO Technical Report Series, No. 373; 1967). The committee recommends that biochemical and metabolic studies be substituted sometimes for the more usual toxicological studies when the effects of food additives are evaluated. If the additive is completely broken. down in normal metabolism to common dietary or body constituents, toxicological investigation is a wasted effort, for the additives are then behaving like foods. For such cases an acceptable daily intake has been calculated such that the additive should not increase the food component into which it is converted by more than about 5 per cent of the amount in an average diet. The fatty emulsifiers such as acetic, citric and lactic acid and fatty acid esters of glycerol are in this category, for in metabolism they are all hydrolysed to acceptable dietary constituents.

The committee says that toxic concentrations of arsenic, lead or heavy metals are unlikely to be found in food now, but it gives specifications to encourage the use of good quality raw materials and equipment. Most normal diets probably supply $1 \cdot 5-2 \cdot 0 \mathrm{mg}$ of arsenic each day. The committee puts the maximum acceptable load at $0.05 \mathrm{mg} / \mathrm{kg}$ of body weight, and recommends that the maximum content of arsenic in food additives be $3 \mathrm{mg} / \mathrm{kg}$; for heavy metals they recommend $40 \mathrm{mg} / \mathrm{kg}$, and for lead $10 \mathrm{mg} / \mathrm{kg}$ in cases where the daily intake of the additive exceeds $1 \mathrm{~g}$.

In several cases a decision on the use of an additive has had to be deferred because of lack of data. Of the natural stabilizers, furcelleran and its salts could not be evaluated in the absence of toxicological data. Concerning carrageen and its salts, now used as food additives, toxicological data are inadequate to establish an acceptable daily intake, and the committee recommends that this information be made available within the next four years so that a decision may be taken on permission to continue the use of this substance. Information is also inadequate concerning those long established additives gum arabic, karaya, tragacanth and carob bean gum, although tentative specifications have been prepared. Details of all specifications will be given in monographs containing biological data and toxicological evaluation of the various additives, and the committee suggests that a decision to use a particular additive should only be taken after consulting the detailed monographs.

\section{Making Air Fresh}

"TAKE a deep breath and be sick" is a wry comment sometimes heard in the United States. In Britain, 